\title{
STRUCTURED EVALUATION OF PRE-CONSTRUCTION COST ALTERNATIVES WITH BIM AND RESOURCE INTEGRATED SIMULATION
}

\author{
Gulsum Sevde Baltasi ${ }^{1}$, Ragip Akbas ${ }^{2}$
}

\begin{abstract}
Despite increasing use of BIM and automation technologies in construction, reliable estimation and management of project costs is still a challenge for contractors. Although contractors are able to schedule and plan their project costs with the current estimating and planning tools during estimation stage, most analysis approaches that compare resource, sequence and method alternatives during pre-construction remain manual and based on experience. This limitation frequently causes contractors not to consider cost-efficient alternatives and even to lose a bid. Furthermore, the reasoning behind these decisions is not properly captured for use later in the project or future projects. Even with BIM use, 4D/5D tools primarily visualize the project with pre-determined cost and time information rather than using a simulation model. This paper shows a structured alternative comparison approach on a BIM and resource integrated discrete-event simulation platform (GSimX) to improve project cost analysis. With this approach, the simulation model on BIM helps compare possible crew, equipment, and other resource alternatives by time and cost while satisfying method, capacity, and spatial constraints. We demonstrate how contractors can improve pre-construction planning with structured pre-construction alternative evaluation on the geometryintegrated planning approach of GSimX.
\end{abstract}

Keywords: Construction simulation, BIM, pre-construction planning, resource management.

\section{INTRODUCTION}

Competitive conditions make it harder for contractors to gain new projects. Making reliable estimates during tender and pre-construction stages is vital for contractors to survive. Even after a contractor is awarded a project, cost issues remain as a major problem. It is well established that decisions taken prior to construction, either during tender or pre-construction stage, has high impact for costs during the project execution.

Use of BIM tools increasingly improve planning and estimation during preconstruction stages and allows contractors to estimate project costs, visualize schedule and evaluate how overall project costs are distributed. However, use of BIM during preconstruction is yet to reach its potential. With improved automation technologies in construction projects, it became possible to see the effects of selection

1 Master of Science Candidate, Graduate School of Science, Engineering and Technology, Construction Management Division, Istanbul Technical University, and Research Assistant, Ozyegin University, Istanbul, Turkey, sevde.baltasi@ozyegin.edu.tr

2 Assistant Professor, Department of Civil Engineering, Ozyegin University, Istanbul, Turkey, ragip.akbas@ozyegin.edu.tr 
and usage of some cost parameters, like construction equipment such as tower cranes (Irizarry and Karan 2012). On the other end, cost management approaches are extended to use BIM, sometimes called 5D, by combining BIM models with schedule with cost parameters (Smith 2014). However, there is lack of general purpose automated tools to evaluate process alternatives with BIM early in the project.

In practice, pre-construction alternative analysis is commonly conducted using manual or semi-automated approaches by experts in companies, although potential cost impact of alternatives is very significant. Experts decide on the number and type of equipment based mostly on previous work data and experience, and perform comparisons typically focusing on one major resource at a time. This leads to a biased and limited evaluation of alternatives. As such, existing approaches and technologies are insufficient for easily defining resources, their allocations and work sequences to evaluate alternatives.

We aim for a simple and natural simulation approach that can generate project plan alternatives using BIM data and evaluate results in terms of cost, time and resource performance. We focus particularly on evaluation of resource and method alternatives for residential and commercial buildings during pre-construction with existing architectural design. Pre-construction alternative comparison helps contractor see the cost and time effects of options in tender stage to build a master plan for the project and generate a more reliable estimate. The options include any change in workflow sequence, number, type and location of equipment, and crew type and quantity to evaluate cost and time impact. Moreover, through sensitivity analysis, planners can focus on most important cost factors.

This study uses a BIM and resource integrated planning and simulation prototype software called GSimX to perform pre-construction cost analysis. The software simulates actions of crews, equipment and other resources on 3D BIM, satisfying construction method, capacity and spatial constraints, and generating cost, time and performance results for alternatives through a combination of discrete-event and agent-based simulations. We extend the application for structured generation and evaluation of simulation alternatives considering equipment and crew related parameters.

\section{BACKGROUND}

As common industry practice, pre-construction alternative analysis is conducted by contractors to determine an overall plan, required resources and overall cost for the project. Planners extract information from company previous projects and combine them with their knowledge and experience to aid for this process (Waly and Thabet 2003). Specifics for major resources, especially types of equipment are selected based on past data and project requirements. In addition, number of crews for a new project is mainly determined by relative size and similarity of finished projects. Crew and equipment productivity is also assigned based on past project productivity rates, which may change depending on site conditions and weather. Such a decision-making process is limited by the knowledge and expertise area of the managers and decision makers, and even an expert may focus on and produce alternative cost analysis for major items, not the whole project.

To aid preconstruction planning, Critical Path Method (CPM) based scheduling tools are traditionally used with project quantities and company cost database to generate an 
estimate. However, such estimations are limited as they are built after decisions on resource usage, construction method and sequence. It is hard to reflect realistic activity relations with an already defined project sequence and easily generate alternatives. Additionally, equipment and crew assignments do not reflect behaviors and resource flows. An equipment is typically considered to be assigned to an activity for its whole duration, although equipment behavior is more complex in practice and rely on many external input including available space for equipment, equipment reaching capacity due to obstacles. All these can impact project sequence, work method, and therefore time and cost.

To overcome these limitations and to better evaluate resource usage and allocation for estimation, contractors use BIM models to support planning. BIM is commonly used to support CPM based planning and estimation tools, such as a visualization tool combined with the preconstruction schedule as 4D CAD model (Koo and Fischer 2000). This lets contractors see any visual conflict prior to execution to make necessary adjustments. Although it brings some advantages like resolving possible conflicts, these approaches do not improve on alternative evaluation during preconstruction. It is far from full potential of BIM in aiding the planning process.

Simulation is a common alternative evaluation technique for construction since it helps see the impact of a change either on local parts or on the whole system. With simulation, it is possible to model and analyze effect of any change on overall project. Simulation techniques provide planners ways to predict operations performance for resource selection and allocation for improved productivity (Cheng and Feng 2003). In construction industry, simulation has long been used for resource optimization (Chen et al. 2012; Siu et al. 2015). However, these approaches remain limited when considering many resources and complex 3D interactions within a project. While it is possible to build a resource-based model for simulation by changing and selecting the appropriate equipment with existing simulation models with focus on a particular set of operations (Shi and AbouRizk 1997), building a simulation model for a non-trivial project, evaluating resource quantity and type alternatives is prohibitively time consuming and prone to errors. In addition, including spatial limitations and adding behavior to resources is not possible for complex projects. Although existing research generates simulation models from product models or BIM (Tommelein et al. 1994), building simple alternatives while reflecting resource behavior is a challenge.

In summary, although existing approaches support various elements of preconstruction cost evaluation, there is a lack of a simple approach for alternative analysis for the overall project on BIM in a structured way, evaluating primary resources throughout construction.

\section{RESEARCH METHOD}

To be able to compare and analyze alternatives within the scope of this study, we use a novel resource-integrated simulation software prototype, called GSimX (Akbas 2016; Baltasi and Akbas 2016). This software implements a new construction simulation approach which allows planners to directly describe construction process including naturally defined resources within a BIM environment. In GSimX simulation, construction resources act on planning structures built directly on building element groups, called location flows. Location flows relate activities with BIM elements to form queueing networks to be analyzed during simulation (Akbas 2003). GSimX implements 
a hybrid simulation approach combining discrete-event simulation with agent-based simulation. Discrete-event simulation manages the overall project simulation, while behavior of crews and major equipment are simulated through agent-based simulation, which includes spatial interactions in 3D and resource capacities. GSimX also has integrated resource management, maintaining resources in pools and managing allocations. Work cannot proceed if any required resource is not available.

Unlike most traditional simulation approaches that visualize results on BIM afterwards, GSimX simulates activities and resources directly on BIM. GSimX imports BIM data and project schedule from widely used software. Planners can define primary resources and their behavior within GSimX. The optional input schedule helps define the overall planning structure and relationships, while all elements of construction is simulated considering resource capability and availability, spatial considerations, and method sequences. Users can generate different alternatives by changing parameters for crew and equipment entities, work sequences, resource allocation to each individual activity, to evaluate cost and time impact. Simulation results can be obtained as 3D visualization, charts, detailed outputs of each individual alternative and as a summary.

In this study, our goal is to provide a simple way to perform pre-construction cost analysis on BIM, evaluating multiple parameters to provide solutions at the project level. Rather than trying to optimize detailed parameters, which is hard early in a project, we focus on evaluating important high-level parameters.

To realize the objective of early-stage structured alternative evaluation, we added several functionalities to GSimX. First, we added detailed cost information for resources (Baltasi and Akbas 2016). Second, we provided simple ways to automatically generate alternatives. Primary cost factors we consider for project alternatives are labor, equipment and indirect costs. Crew cost depends on its labor composition and their standard and overtime hourly costs. Each equipment is assigned an hourly rate including fuel and operator cost if owned or a monthly cost if rented. For indirect cost, a daily fixed rate is assigned. Material cost is not taken into consideration since we assume alternatives do not change the design and affect the bill of quantities for materials for 3D BIM.

With GSimX, structured simulation runs with various parameter options result in various cost and time solutions. While pre-construction alternatives are quite varied, we focus on resources and work sequences. After each simulation, cost and duration for the overall project, costs over time for each individual resource, and other important resource factors such as utilization and idle times are obtained. With the proposed approach, a structured alternative comparison will allow contractors to simulate all resources for the whole project and evaluate performances to choose the most suitable alternative.

\section{TEST CASE}

We demonstrate structured alternative comparison using GSimX on a synthetic test case of a construction project with two four story office buildings (Figure 1). The test case is designed to be simple and realistic, while being sensitive to labor, equipment, and work sequence changes. Two buildings are close enough to share a tower crane located between the buildings. The analysis focuses on structural (reinforced concrete) and architectural works. Structural activities are broken into column, wall, and floor 
activities. Following these, major architectural activities include installing gypsum walls, insulation, plaster, and painting.

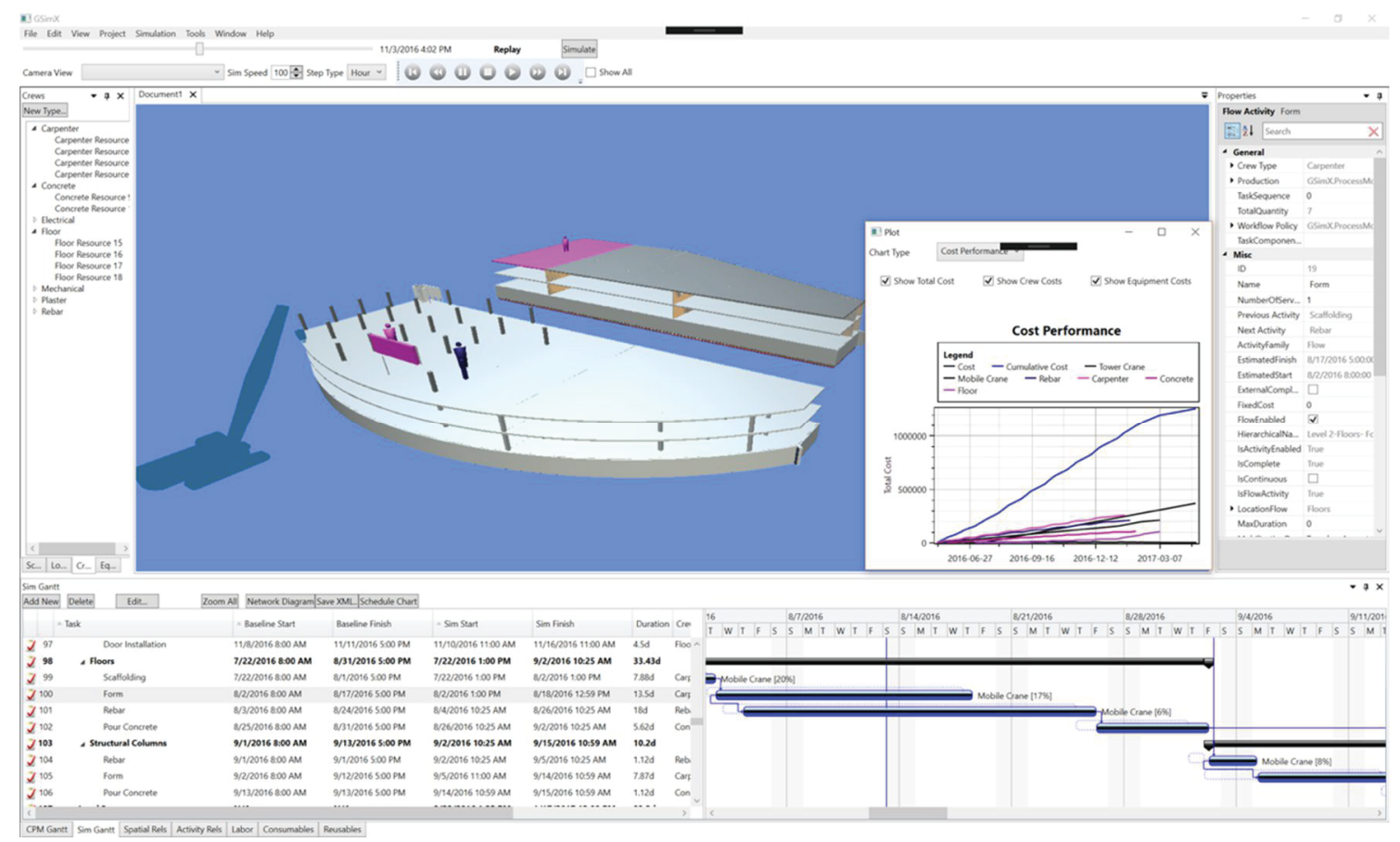

Figure 1: A screenshot for the test case in GSimX

First, we built the simulation model in GSimX, starting by importing the buildings modelled in Autodesk Revit, and master schedule defined in Microsoft Project into GSimX. The duration of construction activities is calculated based on the mean productivity rates gathered by examining published man-hour calculations, together with experts from private sector. For given crew compositions, production rates are obtained for crews acting on each activity.

Afterwards, location flows were defined in GSimX by linking related scopes of activities with BIM entities to install. Next, resources are added in GSimX. Crew types used in this study were carpenter, rebar, concrete, floor and plaster. Primary major equipment used in the test case are mobile crane(s) or a tower crane. Cranes are defined with specific inner and outer operating radius they can access. During simulation, mobile cranes can move next to actual work location, while tower cranes are at a fixed location. Next, required resources for each activity are defined, primarily as type of crew and equipment. Equipment assignment can also be as a percentage value reflecting the percent of equipment time allocated when in use.

For cost calculation, we assigned hourly rates for equipment and labor for crews. We assume that crews only add to the project cost when they are in use. For example, if two crews out of four available total are used on a particular day, only labor for those two crews is charged. We also added a fixed indirect daily cost.

Once the simulation model is ready in GSimX, we built simulation scenarios and compared several categories of alternatives for the test case. Evaluated alternative resource parameters and their quantities are summarized in Table 1. The base case scenario has three mobile crews in total and four available crews for all trades except for concrete (two). Two major crane types are possible for activities: either one tower crane or one to three mobile cranes. Alternatives for number of crews are specified after crane 
type and quantity. Numbers of rebar, concrete and carpenter crews are varied to form alternatives. In addition, alternatives were generated for number of crews per activity. Two or more crews can work simultaneously on an activity scope, possibly starting from opposite ends.

Table 1: Evaluated resource alternatives for simulation

\begin{tabular}{ccc}
\hline Parameter & Alternative & Quantity \\
\hline Equipment type & Tower crane / Mobile crane & $1 / 1-3$ \\
Number of crews per trade & Rebar and carpenter crews & $1-4$ \\
Crews per activity & Rebar and formwork activities & $1-2$ \\
\hline
\end{tabular}

After each alternative simulation, total cost, category costs and project duration is recorded and results are evaluated. First, alternatives evaluated for different equipment type and quantities are compared to the base case (Figure 2). Results show that using mobile crane instead of tower crane results in shorter project duration and lower total cost, and two mobile cranes deliver the best scenario.

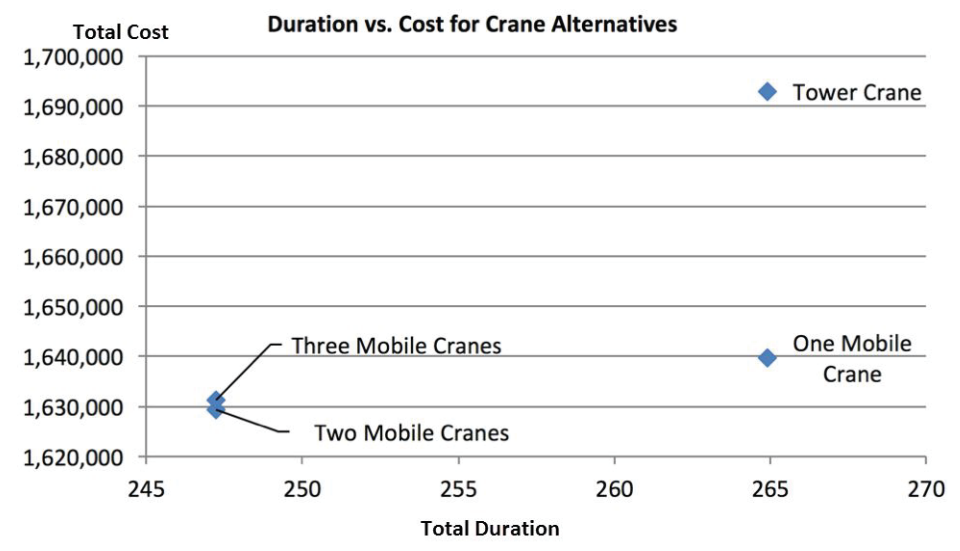

Figure 2: Project Duration vs. Cost for Crane Alternatives

Next, alternatives were formed for varying number of crews per trade for each equipment alternatives and project duration and total costs were evaluated. (Figure 3). Results show that using two crews per activity with multiple mobile cranes is preferable and having two rebar crews leads to the best evaluated alternative. Lastly, effect of number of crews per trade on cost and duration is investigated (Figure 4), which show that the parameter that is most sensitive for cost variance is carpenter crew followed by rebar crew. In addition, when single crew per activity is used, cost and time effect of reducing rebar and carpenter crews is negligible. By examining charts, planners can decide on the right crew and equipment quantity and see the effects of any change on any crew. 

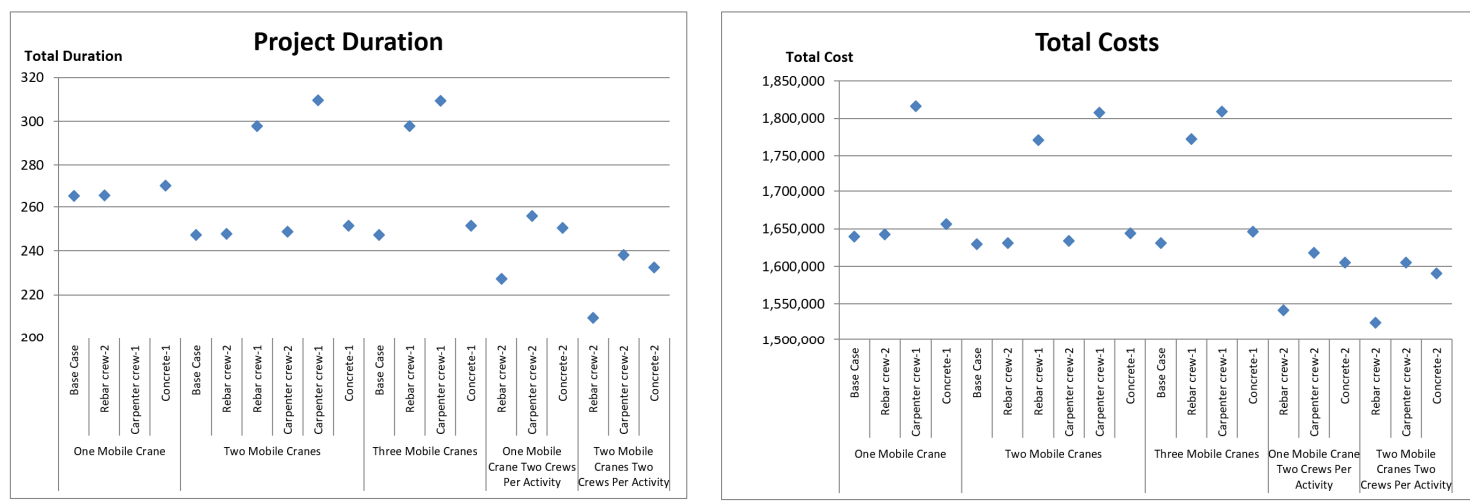

Figure 3: Total cost for different number of crews both on trade and on activity for varying number of mobile cranes
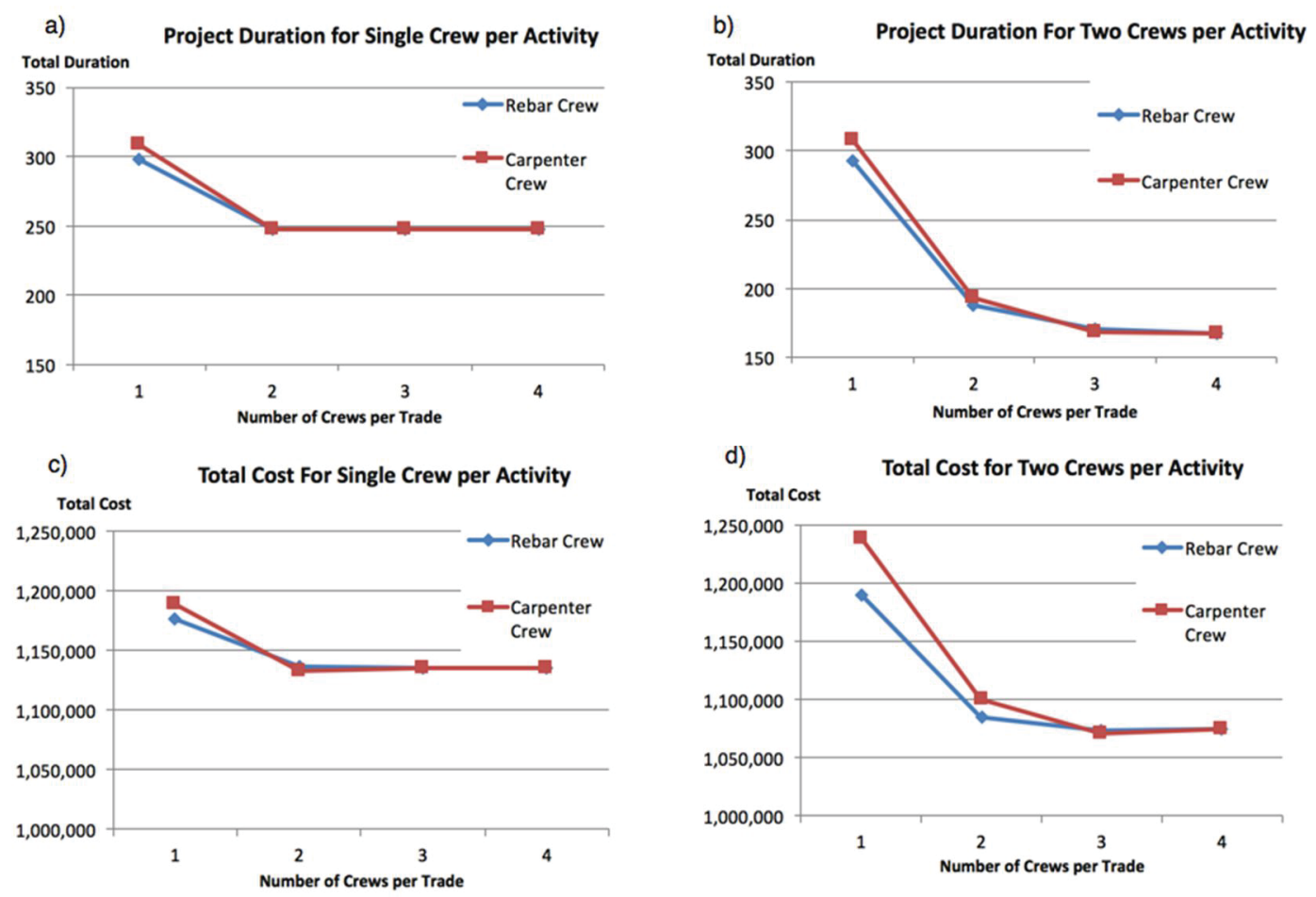

Figure 4: Effect of number of crews per trade on project duration and cost

This test case demonstrates that, in addition to being integrated with resources and BIM, comparing alternatives with GSimX is quicker and easier compared to traditional approaches. Changing all simulation parameters and simulating new scenarios is a simple user operation. For this small realistic test case with 1248 entities and 156 activities, simulating while simultaneously visualizing in 3D, takes less than a minute on a modern notebook computer.

\section{CONCLUSIONS}

We have described an approach for pre-construction resource alternative analysis on BIM using a prototype simulation software called GSimX. We have demonstrated easy evaluation of time and cost results for major resource decisions including equipment type and quantity, number of crews per trade, and number of crews per activity. In 
addition, based on simulated scenarios, we described ways to choose the most suitable alternative and perform sensitivity analysis for trades in terms of manpower. These major resource decisions early in the project are rarely considered in an integrated way. Using such an alternative comparison approach on BIM can help contractors create better estimates supporting project goals.

This study is an ongoing research for more general alternative analysis during preconstruction planning. We plan to apply simulation optimization techniques to automatically evaluate and pick alternatives. While analysis here is deterministic, GSimX supports stochastic parameters, which will facilitate project risk analysis.

\section{REFERENCES}

Akbas, R. (2003). Geometry-based Modeling and Simulation of Construction Processes. Ph.D., Stanford University, Stanford, CA.

Akbas, R. (2016). BUILD-CYCLE: Integrated analysis and visualization for advanced construction planning. European Energy Innovation, Prologue Media Ltd, Hertfordshire, UK, 46.

Baltasi, G. S. and Akbas, R. Alternative comparison for estimating and pre-construction analysis using geometry-integrated planning and simulation. Proc., ACE 2016: 12th Int. Conference on Advances in Civil Engineering.

Chen, S.-M., Griffis, F. H., Chen, P.-H. and Chang, L.-M. (2012). Simulation and analytical techniques for construction resource planning and scheduling. Automation in Construction, 21, 99-113.

Cheng, T.-m. and Feng, C.-w. (2003). An effective simulation mechanism for construction operations. Automation in Construction, 12(3), 227-244.

Irizarry, J. and Karan, E. P. (2012). Optimizing location of tower cranes on construction sites through GIS and BIM integration. ITCON, 17, 351-366.

Koo, B. and Fischer, M. (2000). Feasibility Study of 4D CAD in Commercial Construction. Journal of Constr Eng and Management, 126(4), 251-260.

Shi, J. and AbouRizk, S. (1997). Resource-based modeling for construction simulation. Journal of Constr Eng and Management, 123(1), 26-33.

Siu, M.-F. F., Lu, M. and AbouRizk, S. (2015). Resource Supply-Demand Matching Scheduling Approach for Construction Workface Planning. Journal of Constr Eng and Management, 142(1).

Smith, P. (2014). BIM \& the 5D Project Cost Manager. Procedia - Social and Behavioral Sciences, 119, 475-484.

Tommelein, I. D., Carr, R. I. and Odeh, A. M. (1994). Assembly of Simulation Networks Using Designs, Plans, and Methods. Journal of Constr Eng and Management, 120(4), 796.

Waly, A. F. and Thabet, W. Y. (2003). A virtual construction environment for preconstruction planning. Automation in construction, 12(2), 139-154. 